\title{
A general form of Drucker-Prager type smooth and convex plastic potential. Part 1: Definition and features
}

\author{
Aleksander Szwed $^{1, *}$, and Inez Kamińska ${ }^{1}$ \\ ${ }^{1}$ Warsaw University of Technology, FCE, ul. L. Kaczyńskiego 16, 00-637 Warszawa, Poland
}

\begin{abstract}
A general form of Drucker-Prager type plastic potential suitable for concrete and soil is proposed, allowing to distinguish qualitative difference between stress states with prevailing tension from those with dominant compression. The potential includes six material constants and one arbitrarily chosen function $g$ describing deviatoric section of the potential surface. The influence of the parameters on the shape of the considered yield surface is discussed. The verification of convexity of the proposed potential is carried out in order to impose conditions on the parameters and the unknown function $g$. The calibration based on results of typical tests for concrete presented in the literature is proposed and the values of the coefficients are given out in relation to the yield limit in uniaxial compression test for common proportions of yield limits for other basic tests.
\end{abstract}

\section{Introduction}

The Drucker-Prager potential [1] is widely used for modelling plasticity in concrete and soil. It determines an appropriate first approximation of the materials' behaviour, allowing to distinguish the stress states with predominant compression from the stress states with prevailing tension. Its simplicity is an advantage if any rough analysis of construction is performed. On the other hand, it cannot fully describe complex behaviour of concrete when used in yield condition for two main reasons. The deviatoric section of the Drucker-Prager potential is circular, while for concrete it is approximately triangular for low values of hydrostatic pressure and approaches a circle for increasing compressive stress [2]. Also, according to experimental results $[3,4]$, the slope of meridians changes with hydrostatic stress, while for the Drucker-Prager function the slope is fixed.

Functions used in yield conditions and flow rules are assumed to be convex functions of stress tensor [5, 6]. Also, in order to avoid computational problems, they are differentiable in all their domain (they are potentials). Both these attributes are convenient when solving a problem of plasticity and comply with results of tests performed on various materials.

In this paper, a general form of convex and smooth yield function is proposed, where two Drucker-Prager cones are used as an envelope. To properly describe the characteristic

\footnotetext{
* Corresponding author: a.szwed@il.pw.edu.pl
} 
features of behaviour of concrete, a modification of deviatoric section is introduced, using a classical shape function proposed by Podgórski $[7,8]$. Then basic attributes of the potential are discussed, along with the choice of parameters and comparison to experimental results. The gradient of the function is calculated and a proof of convexity is carried out.

\section{Definition of potential}

\subsection{Envelope of potential surface}

Plastic potentials are functions of invariants of the stress tensor. A set of three independent invariants can be chosen arbitrarily. In this paper the following cylindrical invariants are considered:

$$
\xi=\frac{1}{\sqrt{3}} \operatorname{tr} \boldsymbol{\sigma}, r=\sqrt{\operatorname{tr} \mathbf{s}^{2}} \text { and } \theta=\frac{1}{3} \arccos \left[\frac{\sqrt{6} \operatorname{tr} \mathbf{s}^{3}}{\sqrt{\operatorname{tr}^{3} \mathbf{s}^{2}}}\right],
$$

where $\mathbf{s}$ is the deviator of stress tensor $\boldsymbol{\sigma}$ and $\operatorname{tr}$ denotes operation of trace of a tensor. For the purpose of clarity, the Haigh-Westergaard [9] coordinate system $\left(\sigma_{1}, \sigma_{2}, \sigma_{3}\right)$ is used to illustrate potential surfaces and their cross-sections, see Figure 1.

The Drucker-Prager function [1] is described as follows:

$$
f_{D P}(\xi, r)=\xi+\alpha r-\beta \quad \text { for } \quad r \geq 0
$$

where $\alpha$ and $\beta$ are material parameters, which are calculated using results of two chosen experiments, typically uniaxial compression test and uniaxial tension test. The DruckerPrager cone $f_{D P}(\xi, r)=0$ is shown in Figure 1.
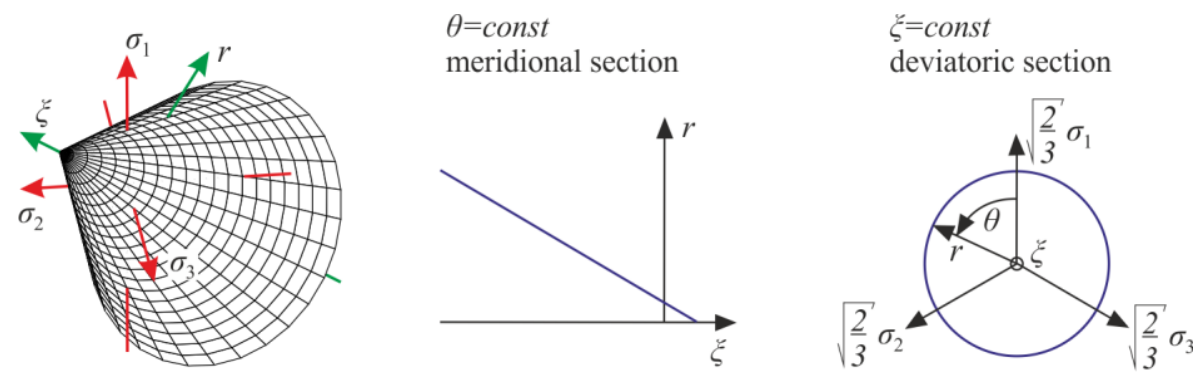

Fig. 1. Drucker-Prager yield surface in Haigh-Westergaard coordinate system.

As mentioned above, the slope of the meridian (i.e. a cross-section of potential surface with $\theta=$ const ) should evolve with lowering $\xi$. In order to acquire this feature, a surface containing two Drucker-Prager cones is adopted as an envelope of a new potential surface:

$$
f_{E N V}(\xi, r)= \begin{cases}\xi+\alpha_{1} r-\beta_{1}=0 & \text { for } \quad 0 \leq r \leq r_{P}=\frac{\beta_{2}-\beta_{1}}{\alpha_{2}-\alpha_{1}} \\ \xi+\alpha_{2} r-\beta_{2}=0 & \text { for } \quad r>r_{P},\end{cases}
$$

where $0<\alpha_{1} \leq \alpha_{2}$ and $\beta_{1} \leq \beta_{2}$. 


\subsection{Definition of potential}

The proposed smooth and convex potential is constructed as follows. Initially, an axisymmetric potential surface of second order is built based on (3). Then, function $g(\theta)$ is introduced to modify the deviatoric cross-section. As a result, one of the meridians of the potential surface (in this case, as calibrated below: the tension meridian $\theta=0$ ) is a smooth approximation of the corresponding meridian of (3) and the deviatoric section is described by scaled $g(\theta)$.

The proposed form of function is:

$$
f(\xi, r, \theta)=\xi-F(r, \theta)
$$

where:

$$
\begin{gathered}
F(r, \theta)=\frac{f_{1 N}(r, \theta)+f_{2 N}(r, \theta)-P(r, \theta)}{2} \\
f_{1 N}(r, \theta)=\beta_{1}-\alpha_{1} \sqrt{A^{2}+\left[\frac{r}{g(\theta)}\right]^{2}}, \quad f_{2 N}(r, \theta)=\beta_{2}-\alpha_{2} \sqrt{A^{2}+\left[\frac{r}{g(\theta)}\right]^{2}} \\
P(r, \theta)=\sqrt{B^{2}+\left[f_{1 N}(r, \theta)-f_{2 N}(r, \theta)\right]^{2}}
\end{gathered}
$$

and $0<\alpha_{1} \leq \alpha_{2}, \beta_{1} \leq \beta_{2}, A>0, B>0$ are material constants. Shape function $g(\theta)$ is a positive function of the Lode angle. The further restriction on its form is due to convexity and it is derived in Section 3. The subsequent yield condition is defined as:

$$
f(\xi, r, \theta)=\xi-F(r, \theta)=0 .
$$

The meridians of surface (6) are shown in Fig. 2. The envelope for a chosen meridian $\theta=$ const consists of two lines:

$$
\xi_{1}(r, \theta)=-\frac{\alpha_{1}}{g(\theta)} r+\beta_{1} \quad \text { and } \quad \xi_{2}(r, \theta)=-\frac{\alpha_{2}}{g(\theta)} r+\beta_{2} .
$$

Parameters $\alpha_{1}$ and $\alpha_{2}$ control the slopes of meridians.
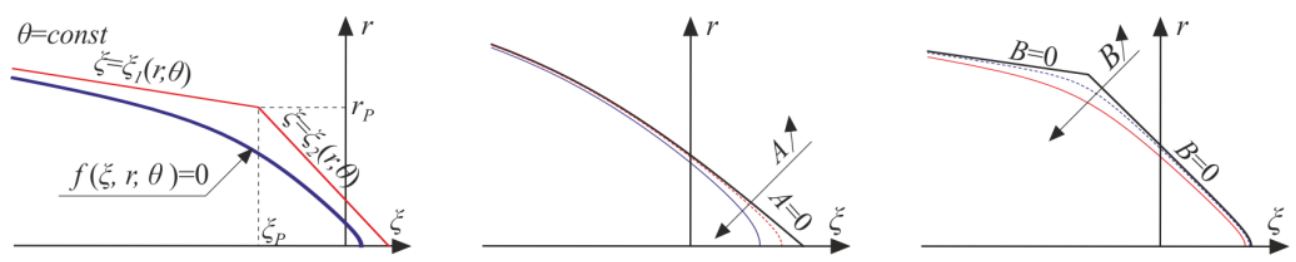

Fig. 2. Meridional sections of potential surface (6).

Constant $A$ determines the shape of the apex of (6). In case of $A=0$ the function is nondifferetiable for $r=0$, while its increase causes the increase of radius of curvature near the tip, see Figure 2. Parameter $B$ is responsible for the curvature near the transition zone between the envelope lines (the area around $r=r_{P}$ ). For $B=0$ an edge appears in the yield surface, but with increasing $B$ the transition zone smoothens, see Figure 2.

In case of $A=0, B=0, \alpha_{1}=\alpha_{2}=\alpha, \beta_{1}=\beta_{2}=\beta$ and $g(\theta) \equiv 1$ the proposed potential reduces to Drucker-Prager function (2). 


\section{Differentiability and convexity}

To show that the function is differentiable its gradient is obtained. The components of the gradient vector in the cylindrical coordinate frame $(\xi, r, \theta)$ (see also Fig. 1) are as follows:

$$
\begin{aligned}
& \operatorname{grad} f=\frac{\partial f}{\partial \boldsymbol{\sigma}} \rightarrow\left[\begin{array}{lll}
f_{, \xi} & f_{, r} & \frac{1}{r} f_{, \theta}
\end{array}\right]^{T}=\left[\begin{array}{lll}
1 & \frac{f_{3} r}{g Q P} & -\frac{f_{3} g^{\prime} r}{g^{2} Q P}
\end{array}\right]^{T}, \\
& \text { where: } f_{3}=\alpha_{1} f_{2 N}+\alpha_{2} f_{1 N}-\left(\alpha_{1}+\alpha_{2}\right) F, \quad Q=\sqrt{(A g)^{2}+r^{2}}
\end{aligned}
$$

In this Section, arguments of all functions are omitted for clarity of the equations.

The first two components of (8) are continuous for $A>0, B>0$ and continuous functions $g, f_{1 N}$ and $f_{2 N}$. The polar component of the gradient is unique if $g$ is a differentiable function. For $r=0$ the gradient is the unit vector coincident with $\xi$ axis.

To ensure that function (4) is convex, the nonnegativeness of the following curvature tensor must be proved [10]:

$$
\boldsymbol{\kappa} \rightarrow\left[\begin{array}{ccc}
f_{, \xi \xi} & f_{, \xi r} & \frac{1}{r} f_{, \xi \theta} \\
f_{, \xi r} & f_{, r r} & \left(\frac{1}{r} f_{, \theta}\right),_{r} \\
\frac{1}{r} f_{, \xi \theta} & \left(\frac{1}{r} f_{, \theta}\right),_{r} & \frac{1}{r^{2}} f_{, \theta \theta}+\frac{1}{r} f_{, r}
\end{array}\right] .
$$

A matrix is nonnegative if all its principal minors are nonnegative [11]. For the proposed function $f_{, \xi \xi}=0, f_{, \xi r}=0$ and $f_{, \xi \theta}=0$, so for (4) to be convex the following inequalities have to be fulfilled:

$$
\begin{gathered}
D_{1}=f_{, r r}=\frac{A^{2} g f_{3}}{P Q^{2}}+\frac{\left(\alpha_{2}-\alpha_{1}\right)^{2} B^{2} r^{2}}{2 g^{2} P^{3} Q} \geq 0, \\
D_{2}=\frac{1}{r^{2}} f_{, \theta \theta}+\frac{1}{r} f_{, r}=\frac{\left(\alpha_{2}-\alpha_{1}\right)^{2} B^{2} r^{2}\left(g^{\prime}\right)^{2}}{2 g^{4} P^{3} Q^{2}}+\frac{f_{3}\left(f_{4} Q^{2}+r^{2}\left(g^{\prime}\right)^{2}\right)}{g^{3} P Q^{3}} \geq 0, \\
D_{3}=D_{1} D_{2}-\left[\left(\frac{1}{r} f_{, \theta}\right),\right]^{2}=\frac{D_{1} f_{3} f_{4}}{g^{2} P Q} \geq 0, \quad \text { where } \quad f_{4}=g^{2}-g g^{\prime \prime}+2\left(g^{\prime}\right)^{2} .
\end{gathered}
$$

Since functions $P, Q$ and $g$ are positive, therefore to satisfy (10) it is sufficient to fulfil $f_{4} \geq 0$ and $f_{3} \geq 0$. The last function can be rewritten as:

$$
f_{3}=\left(\alpha_{2}-\alpha_{1}\right)\left(f_{2 N}-f_{1 N}+\sqrt{B^{2}+\left(f_{1 N}-f_{2 N}\right)^{2}}\right)+\alpha_{1} P,
$$

which is clearly positive for $0<\alpha_{1} \leq \alpha_{2}$ and $\beta_{1} \leq \beta_{2}$. The other condition has to be satisfied with appropriate choice of shape function $g$.

As shown above, function (4) is differentiable and convex in its domain when $0<\alpha_{1} \leq \alpha_{2}, \quad \beta_{1} \leq \beta_{2}, A>0, B>0$ and $g(\theta)$ is positive and twice differentiable function satisfying the following condition: 


$$
g^{2}-g g^{\prime \prime}+2\left(g^{\prime}\right)^{2} \geq 0 .
$$

In the literature, there are several propositions of the shape functions modifying deviatoric section of yield surface $[6,7,12,13]$. In this paper the following function proposed by Podgórski [7] is used:

$$
g(\theta)=\frac{\cos \left(\frac{1}{3} \arccos (b)-k\right)}{\cos \left(\frac{1}{3} \arccos (b \cos 3 \theta)-k\right)} \text { for } \theta \in\langle 0, \pi / 3\rangle, 0 \leq b<1,0 \leq k \leq \pi / 6 .
$$

It is positive and fullfills (12) for the considered range of $\theta$.

\section{Determination of material constants}

To determine the considered potential the choice of eight material parameters $\left(\alpha_{1}, \alpha_{2}, \beta_{1}\right.$, $\left.\beta_{2}, A, B, b, k\right)$ has to be made.

To calibrate the yield surface results of characteristic tests for concrete are used. The coefficients are calculated considering that the yield surface passes through points representing uniaxial compression test $(\mathrm{C})$, uniaxial tension test $(\mathrm{T})$, equibiaxial compression test (BC) and biaxial compression test where $\sigma_{1}: \sigma_{2}=2$ (BNC). Also, the asymptotes are adjusted to appropriately describe behaviour of concrete qualitatively.

Firstly, the parameters determining envelope lines (7) are obtained. To enable for the yield surface to pass through selected points, the envelope of the tension meridian (3) is constructed as follows. The slopes $\alpha$ are calculated for lines connecting points representing tests $\mathrm{T}, \mathrm{C}$ and $\mathrm{BC}$ on the meridional plane (neglecting the fact that uniaxial compression test does not lay on the tension meridian), see Figure 3:

$$
\alpha_{1}=\frac{\sigma_{C}+\sigma_{T}}{\sqrt{2}\left(\sigma_{C}-\sigma_{T}\right)}, \quad \alpha_{2}=\frac{2 \sigma_{B C}-\sigma_{C}}{\sqrt{2}\left(\sigma_{B C}-\sigma_{C}\right)},
$$

where $\sigma_{C}$ is the plastic limit for uniaxial compression test, $\sigma_{T}$ is the plastic limit for the uniaxial tension test and $\sigma_{B C}$ is the plastic limit for the equibiaxial compression test.

Then, the lines $\xi=-\alpha r+\tilde{\beta}$, passing through $\mathrm{C}$ are translated by distance $d$ forming the asymptotes for the tensile meridian $\xi=-\alpha r+\beta$, where:

$$
\beta_{1}=\frac{\sqrt{2} \alpha_{1}-1}{\sqrt{3}} \sigma_{C}+d \sqrt{1+\alpha_{1}^{2}}, \quad \beta_{2}=\frac{\sqrt{2} \alpha_{2}-1}{\sqrt{3}} \sigma_{C}+d \sqrt{1+\alpha_{2}^{2}} .
$$

The choice of $d>0$ is arbitrary. It influences considerably the course of meridians for moderate values of $\xi$ and it is selected to fit experimental data.

At this point the values of $A$ and $B$ are determined, assuming that the tension meridian is the reference meridian, i.e. $g(0)=1$, and the yield surface includes points representing $\mathrm{T}$ and $\mathrm{BC}$ :

$$
f\left(\frac{1}{\sqrt{3}} \sigma_{T}, \sqrt{\frac{2}{3}} \sigma_{T}, 0\right)=0, \quad f\left(-\frac{2}{\sqrt{3}} \sigma_{B C}, \sqrt{\frac{2}{3}} \sigma_{B C}, 0\right)=0 .
$$




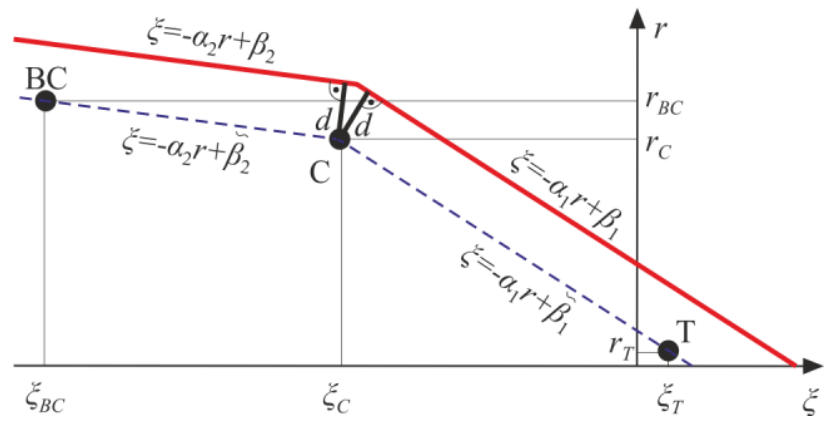

Fig. 3. The choice of parameters $\alpha_{1}, \alpha_{2}, \beta_{1}$ and $\beta_{2}\left(\xi_{T}=\sigma_{T} / \sqrt{3}, \xi_{C}=-\sigma_{C} / \sqrt{3}\right.$, $\left.\xi_{B C}=-2 \sigma_{B C} / \sqrt{3}, r_{T}=\sqrt{2} \sigma_{T} / \sqrt{3}, r_{C}=\sqrt{2} \sigma_{C} / \sqrt{3}, r_{B C}=\sqrt{2} \sigma_{B C} / \sqrt{3}\right)$.

The resultant biquadratic equation for $A$ is as follows:

$$
c_{1}^{2} A^{4}+2\left(\frac{2}{3} c_{1}^{2} c_{3} c_{4}-c_{2} a_{3}^{2}\right) A^{2}+\left(\frac{2}{3} c_{3}^{2}-a_{3}^{2}\right)\left(\frac{2}{3} c_{3}^{2}-a_{4}^{2}\right)=0,
$$

with: $a_{1}=\alpha_{1}\left(\frac{2}{\sqrt{3}} \sigma_{B C}+\beta_{2}\right)+\alpha_{2}\left(\frac{2}{\sqrt{3}} \sigma_{B C}+\beta_{1}\right), a_{2}=\alpha_{1}\left(\beta_{2}-\frac{1}{\sqrt{3}} \sigma_{T}\right)+\alpha_{2}\left(\beta_{1}-\frac{1}{\sqrt{3}} \sigma_{T}\right)$,

$$
\begin{gathered}
a_{3}=\left(\frac{2}{\sqrt{3}} \sigma_{B C}+\beta_{2}\right)\left(\frac{2}{\sqrt{3}} \sigma_{B C}+\beta_{1}\right)-\left(\beta_{2}-\frac{1}{\sqrt{3}} \sigma_{T}\right)\left(\beta_{1}-\frac{1}{\sqrt{3}} \sigma_{T}\right)+\frac{2}{3} \alpha_{1} \alpha_{2}\left(\sigma_{B C}^{2}-\sigma_{T}^{2}\right), \\
c_{1}=a_{1}^{2}-a_{2}^{2}, \quad c_{2}=a_{1}^{2}+a_{2}^{2}, \quad c_{3}=a_{1} \sigma_{B C}+a_{2} \sigma_{T}, \quad c_{4}=a_{1} \sigma_{B C}-a_{2} \sigma_{T} .
\end{gathered}
$$

The solution to (16) is:

$$
\begin{gathered}
A=\frac{1}{c_{1}} \sqrt{c_{2} a_{3}^{2}-\frac{2}{\sqrt{3}} a_{1} a_{2} a_{3} c_{6}-\frac{2}{3} c_{1} c_{3} c_{4}}, \quad B=2 \sqrt{d_{1} d_{2}} \\
\text { where: } \quad c_{5}=\sigma_{B C}^{2}-\sigma_{T}^{2}, \quad c_{6}=\sqrt{3 a_{3}^{2}-2 c_{1} c_{5}}, \\
d_{1}=\frac{2}{\sqrt{3}} \sigma_{B C}+\beta_{1}-\alpha \sqrt{A^{2}+\frac{2}{3} \sigma_{B C}^{2}}, \quad d_{2}=\frac{2}{\sqrt{3}} \sigma_{B C}+\beta_{2}-\alpha_{2} \sqrt{A^{2}+\frac{2}{3} \sigma_{B C}^{2}} .
\end{gathered}
$$

To find the remaining two constants uniaxial compression test and non-equibiaxial compression test are used. The points representing them are located on compression and shear meridian respectively. Parameters $b$ and $k$ are obtained numerically from the following conditions:

$$
f\left(-\frac{1}{\sqrt{3}} \sigma_{C}, \sqrt{\frac{2}{3}} \sigma_{C}, \frac{\pi}{3}\right)=0, \quad f\left(-\frac{\sqrt{3}}{2} \sigma_{B N C}, \frac{1}{\sqrt{2}} \sigma_{B N C}, \frac{\pi}{6}\right)=0,
$$

where $\sigma_{B N C}$ is the plastic limit for the biaxial compression test with $\boldsymbol{\sigma} \rightarrow-\sigma_{B N C} \operatorname{diag}[1,0.5,0]$. For $k=0$ it is possible to find analytical solution for (19) $[14]$.

In case of concrete, the most convenient reference experiment is uniaxial compression test. It is useful to determine all coefficients of the potential, establishing typical 
proportions of the yield limits in relation to reference value $\sigma_{C}: \sigma_{T} / \sigma_{C}=0.1$, $\sigma_{B N C} / \sigma_{C}=1.25, \sigma_{B C} / \sigma_{C}=1.16[15]$ and $\sigma_{T C} / \sigma_{C}=1.25$, where $\eta \sigma_{T C}$ is the yield limit for triaxial compression test where $\boldsymbol{\sigma} \rightarrow-\sigma_{T C} \operatorname{diag}[\eta, 1,1]$ and $\eta=4.91$. Assuming $d=2.75 \sigma_{C}$ the following values of parameters are obtained:

$$
\begin{array}{llll}
\alpha_{1}=0.86424, & \alpha_{2}=5.8336, & \beta_{1}=3.7630 \sigma_{C}, & \beta_{2}=20.462 \sigma_{C}, \\
A=0.14187 \sigma_{C}, & B=16.651 \sigma_{C}, & b=0.98270, & k=0.10489
\end{array}
$$

The value of $d$ was chosen to bring the yield surface near the point representing the triaxial compression test. The resulting yield surface and its sections are shown in Figure 4.
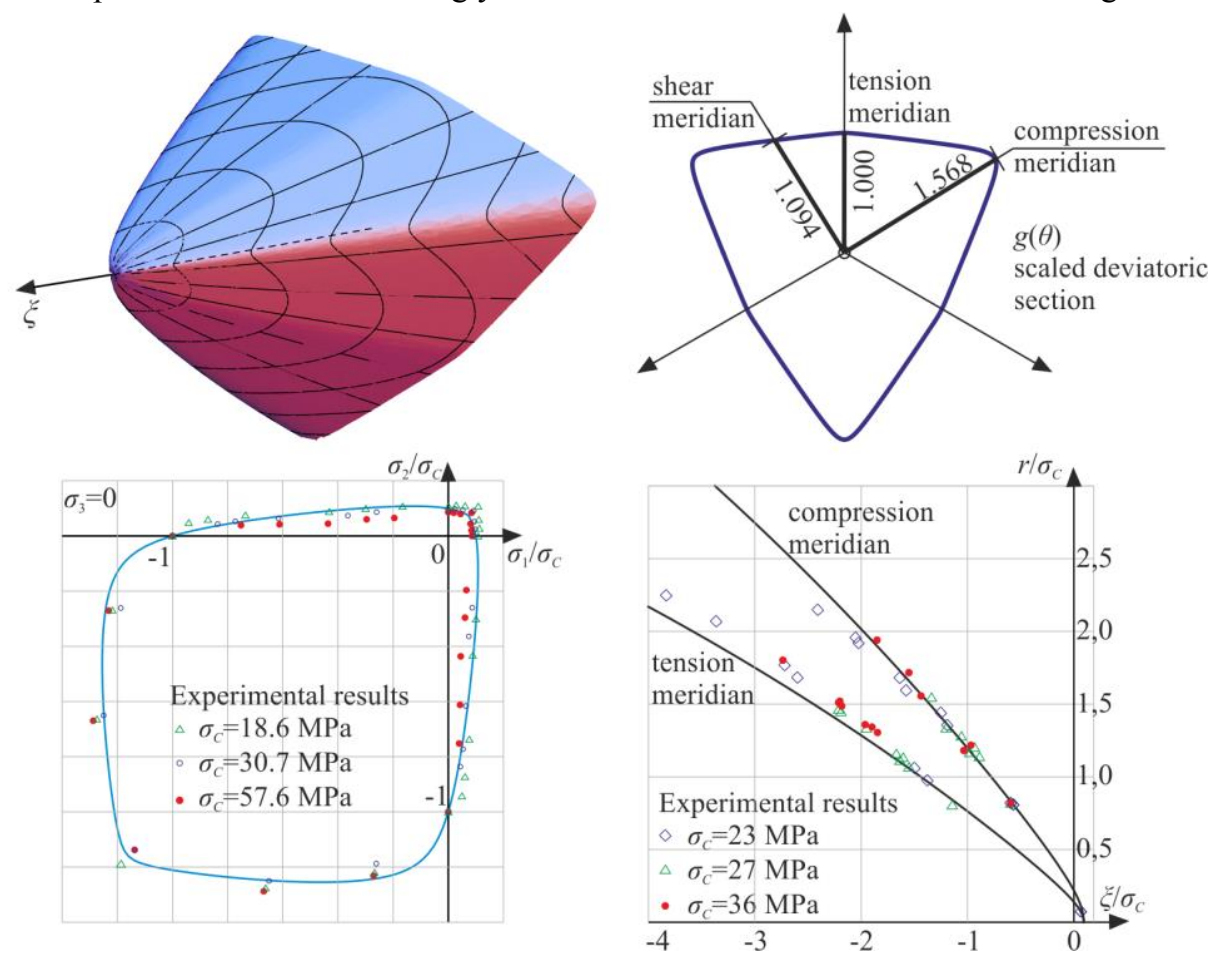

Fig. 4. Yield surface (6) and its deviatoric and meridional sections versus experimental results $[3,15]$.

Potential surface (6) includes majority of the aforementioned general features of the yield surface of concrete with the most important quality: distinction of two zones (tensile and compressive). The meridians are in accordance with the experimental results [3] for small and moderate values of $|\xi|$, which is usually the range of interest observed in civil engineering structures. For very low hydrostatic stress $(\xi \rightarrow-\infty)$, the course of (6) differs from the empirical yield surface, which could be improved by changing envelope $(7)_{2}$ from a straight line to a parabola. Although this change would complicate the form of the potential considerably and it is possible that the gained profit would not justify the increased complexity of computation.

The shape of deviatoric section is close to triangular for all $\xi$. To adjust this inaccuracy, one may assume that coefficients $b$ and $k$ describing function $g$ would depend on $\xi$. 
The plane stress cross-section of the yield surface complies with experimental results [15]. For the tension-tension and tension-compression quadrants, the coincidence between prediction according to (6) and empirical values is very good. It is believed that for compressive behaviour the curve should be more oval, although the deviation implies conservative evaluation of stress state.

\section{Summary}

The considered function establishes the yield surface adequate for describing behaviour of concrete and some types of soil. It enables to distinguish two zones of noticeably different gradients, which is a characteristic feature for quasibrittle materials. Its other main advantages include its smoothness and relatively low complexity, allowing to find simple estimates for the parameters. Also, the conditions for the potential to be convex are sufficiently weak to be able to adjust the yield surface to a wide range of proportions of the yield limits. The plane stress cross-section in compressive quadrant can be further improved by chosing another form of shape function $g(\theta)$.

In Part II [16] of the paper, the potential is used to modify Concrete Damaged Plasticity model [17] for quasibrittle materials. Specifically, the Lubliner yield condition is exchanged by the proposed one.

\section{References}

1. D.C. Drucker, W. Prager, J Appl Mech, 10, 157-165 (1952)

2. N.S. Ottosen, J Eng Mech Div, 103(8), 527-535 (1977)

3. L. Mills, M. Zimmerman, ACI J, 67(10), 802-807 (1970)

4. R. Palaniswamy, S. Shah, J Eng Mech Div, 100(5), 901-916 (1974)

5. J. Lubliner, Plasticity Theory (2005)

6. S. Jemioło, A. Szwed, Budownictwo, 133, 5-51 (OWPW, 1999)

7. J. Podgórski, Ogólny warunek stanu granicznego dla materiałów izotropowych (1983)

8. D. Bigoni, A. Piccolroaz, Int J Solids Struct, 41, 2855-2878 (2004)

9. H.M. Westergaard, J Franklin Inst, 189(5), 553-692 (1920)

10. I. Kamińska, A. Szwed, Sprężystość i lepkosprężystość małych odkształceń, 6, 79-94 (OWPW, 2017)

11. F. Zhang: Matrix Theory. Basic Results and Techniques (Springer, 2011)

12. O.C. Zienkiewicz, G.N. Pande, Finite Elements in Geomechnics, 179-190 (John Wiley \& Sons, 1977)

13. W. Ehlers, Arch Appl Mech, 65, 246-259 (1995)

14. A. Szwed, I. Kamińska, TTS, 12, 129-134 (2017)

15. H. Kupfer, H. Hilsdorf, H. Rusch, ACI J, 66(8), 656-666 (1969)

16. I. Kamińska, Part II - accompanying paper

17. ABAQUS: Theory Manual, Version 6.3 (Hibbit, Karlson \& Sorensen Inc., 2002) 